\title{
AVIAN INFLUENZA VIRUS TRANSMISSION IS SUPPRESSED IN CHICKENS FED LACTOBACILLUS PARACASEI EXPRESSING THE 3D8 SINGLE-CHAIN VARIABLE FRAGMENT PROTEIN
}

\author{
Hoonsung ChOI ${ }^{1}$, Sang In LEE ${ }^{2}$, Shanmugam SureshKumar ${ }^{1}$, Mi-Hyang JEON ${ }^{1}$, \\ Jeom Sun KIM ${ }^{1}$, Mi-Ryung PARK ${ }^{1}$, Kyung-Woon KIM ${ }^{1}$, Ik-Soo JEON ${ }^{1}$, Sukchan LEE ${ }^{3}$ \\ and Sung June BYUN ${ }^{1 *}$ \\ ${ }^{1}$ Animal Biotechnology Division, National Institute of Animal Science, RDA, \\ 1500 Kongjwipatjwi-ro, Iseo-myeon, Wanju-gun, Jeollabuk-do, 55365, Republic of \\ Korea; ${ }^{2}$ Department of Animal Biotechnology, Kyungpook National University, Sangju, \\ Gyeongsangbuk-do, Republic of Korea; ${ }^{3}$ Department of Genetic Engineering, \\ Sungkyunkwan University, Seobu-ro, Jangan-gu, Suwon, Republic of Korea
}

(Received 1 April 2019; accepted 25 September 2019)

The 3D8 single-chain variable fragment ( $\mathrm{scFv}$ ) is a mini-antibody sequence with independent nuclease activity that shows antiviral effects against all types of viruses in chickens and mice. In this study, chickens were treated daily with an oral dose of $10^{9} \mathrm{CFU}$ Lactobacillus paracasei (L. paracasei) expressing either a secreted or anchored 3D8 scFv for three weeks. After L. paracasei administration, the chickens were challenged with avian influenza virus (AIV). From each experimental group, three chickens were directly infected with $100 \mu \mathrm{L}$ of $10^{7.5} \mathrm{EID}_{50} / \mathrm{mL}$ H9N2 AIV and seven chickens were indirectly challenged through contact transmission. Oropharyngeal and cloacal swab samples were collected at 3, 5, 7, and 9 days post-inoculation (dpi) from AIV-challenged chickens, AIV Shedding titres were measured by quantitative real-time PCR. Contact transmission in the chickens that were fed 3D8 scFv-secreting L. paracasei showed a significant reduction in viral shedding when compared with other groups. These results suggest that $L$. paracasei secreting 3D8 provides a basis for the development of ingestible antiviral probiotics with activity against AIV.

Key words: 3D8 scFv, avian influenza virus, Lactobacillus paracasei, oral administration

Probiotics are live microorganisms that can regulate fat metabolism by affecting genes that encode key enzymes (Zhu et al., 2015; Kang et al., 2016). Probiotics can effectively stimulate the immune system (Dec et al., 2015; Patel et al., 2015). Among alternatives to antibiotics in the poultry industry, the use of Lactobacillus as an immune stimulant to enhance nonspecific host defence mechanisms or as an antimicrobial to inhibit bacterial growth has been reported

*Corresponding author; E-mail: pcs1778@korea.kr; Phone: 0082 (63) 238-7263; Fax: 0082 (63) 238-7297 
(Meimandipour et al., 2010; Wang et al., 2013; Ritzi et al., 2014; Kang et al., 2016). The previously described probiotics include therapeutic agents such as ingestible antiviral probiotics that are active against gastrointestinal viral infection (Hoang et al., 2015). Park et al. (2018) have developed a strain of Escherichia coli (E. coli) producing nucleic acid-hydrolysing codon optimised 3D8 single-chain variable fragment (3D8 scFv) for use as a feed additive to prevent norovirus infection (Park et al., 2018). Lactobacillus paracasei (L. paracasei) was shown to express a heavy-chain antibody fragment against rotavirus (Pant et al., 2006).

The 3D8 scFv is an anti-nucleic acid antibody that can bind and hydrolyse nucleic acids without sequence specificity (Kim et al., 2006). 3D8 scFv penetrates cells via the caveolae-lipid raft pathway (Hoffmann et al., 2001). Previous data confirmed that the $3 \mathrm{D} 8 \mathrm{scFv}$ protein suppresses avian influenza virus transmission in transgenic chickens (Byun et al., 2017). In addition, infectious bronchitis virus transmission is suppressed in transgenic chickens expressing the 3D8 $\mathrm{scFv}$ protein (Lee et al., 2019). Hence, $3 \mathrm{D} 8 \mathrm{scFv}$ antiviral activity against various viral infections is broad spectrum, and our study primarily aimed to investigate the prospect of using $L$. paracase $i$ as a delivery system for $3 \mathrm{D} 8 \mathrm{scFv}$ to suppress avian influenza virus in chickens. We confirmed virus shedding in oropharyngeal and cloacal swab samples by quantitative real-time PCR in direct and contact transmission groups. Our findings showed that $L$. paracasei expressing $3 \mathrm{D} 8 \mathrm{scFv}$ could be developed for use as a feed additive to control AIV transmission.

\section{Materials and methods}

\section{Ethics statement}

The challenge study with a live virus was conducted in a biosafety level 2 facility under the supervision of the Institutional Animal Care and Use Committee (IACUC) (2015-111) of Konkuk University, South Korea.

\section{Experimental design, animals and their diets}

Forty conventional healthy specific-pathogen-free (SPF) chickens (threeweek-old) were used in the experiment which lasted for 35 days. Chickens were allocated to four experimental groups and orally administered $10^{9}$ colony-forming units (CFUs) (per chicken/day) of L. paracasei secreting 3D8 scFv (Group 1, G1); L. paracasei anchored 3D8 scFv (Group, G2); L. paracasei (Group 3, G3); control [Group 4 (G4) - phosphate-buffered saline (PBS)] using a syringe and bluntend catheter. Every chicken was individually identified by using tags, and they received water and feed supplied ad libitum. The basal diets were formulated to exceed the nutritional requirements of chickens according to the NRC (1994) recommendations for experimental chickens. 
In vivo AIV infection and transmission studies

After L. paracasei oral administration, we initiated AIV challenge in the treatment groups. Three chickens per group were intranasally challenged with $100 \mu \mathrm{L}$ of $10^{7.5} \mathrm{EID}_{50} / \mathrm{mL}$ H9N2 virus (A/Korean native chicken/Korea/K040110/ 2010). The remaining seven chickens in each group were co-housed in the same containment isolator for $6 \mathrm{~h}$ post-inoculation. To assess viral shedding, oropharyngeal and cloacal swab samples were collected at 3,5, 7, and 9 days postinoculation (dpi) and suspended in $1 \mathrm{~mL}$ of PBS. Suspensions $(200 \mu \mathrm{l})$ were used for RNA extraction using an RNeasy Mini Kit (QIAGEN, Valencia, CA) according to the manufacturer's instructions. The virus shedding values were measured according to Lee et al. (2011). The virus stock was propagated in the allantoic cavity of 10-day-old chicken embryos $(\mathrm{n}=4)$ at $37^{\circ} \mathrm{C}$ for $72 \mathrm{~h}$ according to Spackman et al. (2003). The allantoic fluids were harvested, aliquoted, and stored at $-70{ }^{\circ} \mathrm{C}$ until use. The virus titre in the allantoic fluid was determined based on the $50 \%$ egg infective dose $\left(\mathrm{EID}_{50}\right)$. The $\mathrm{EID}_{50}$ values were measured according to Lee et al. (2011). Viral RNA was extracted from swab samples using the RNeasy kit (QIAGEN, Valencia, CA) according to the manufacturer's instructions. Real-time reverse transcription polymerase chain reaction (RT-PCR) was performed under standard conditions using influenza-specific primers according to Hoffmann et al. (2001) (Fig. 1C). Amplified products of the expected size were purified with QIAquick PCR purification kits (QIAGEN), and nucleotide sequencing was performed (Applied Biosystems, Foster City, CA) according to the manufacturer's instructions (Lee et al., 2011).

\section{Construction of recombinant $\mathrm{L}$. paracasei $p S L P$ - $L D H$ expressing $3 D 8 s c F v$}

The pSLP111.3 expression vector for Lactobacillus, provided by Dr. Jos Seegers, was improved to replace the xylose-inducible promoter with a lactate dehydrogenate (LDH) constitutive promoter (Oozeer et al., 2005). To induce 3D8 scFv protein expression, codon-optimised 3D8 scFv was cloned into pSLPLDH that had been slightly modified from the original vector as previously described (Hoang et al., 2015). 3D8 scFv L. paracasei was anaerobically cultured in de Man, Rogosa and Sharpe medium (Difco Laboratories, Detroit, MI) at $37^{\circ} \mathrm{C}$ for $24 \mathrm{~h}$. To construct the secreted form of 3D8 scFv, the 3D8 scFv gene fused with a protein A tag was inserted downstream of the SlpA secretion signal using the NcoI and AscI restriction enzyme sites. In addition, a stop codon (TAA) was added after the protein A tag to prevent fusion of the 3D8 $\mathrm{scFv}$ with the cell wall-anchoring domain of PrtP (anchor sequence). A cell wall-anchored form of $3 \mathrm{D} 8 \mathrm{scFv}$ was generated by inserting the $3 \mathrm{D} 8 \mathrm{scFv}$ gene using the NcoI and AscI restriction enzyme sites upstream of the anchor sequence according to Hoang et al. (2015) (Fig. 1A). 
A
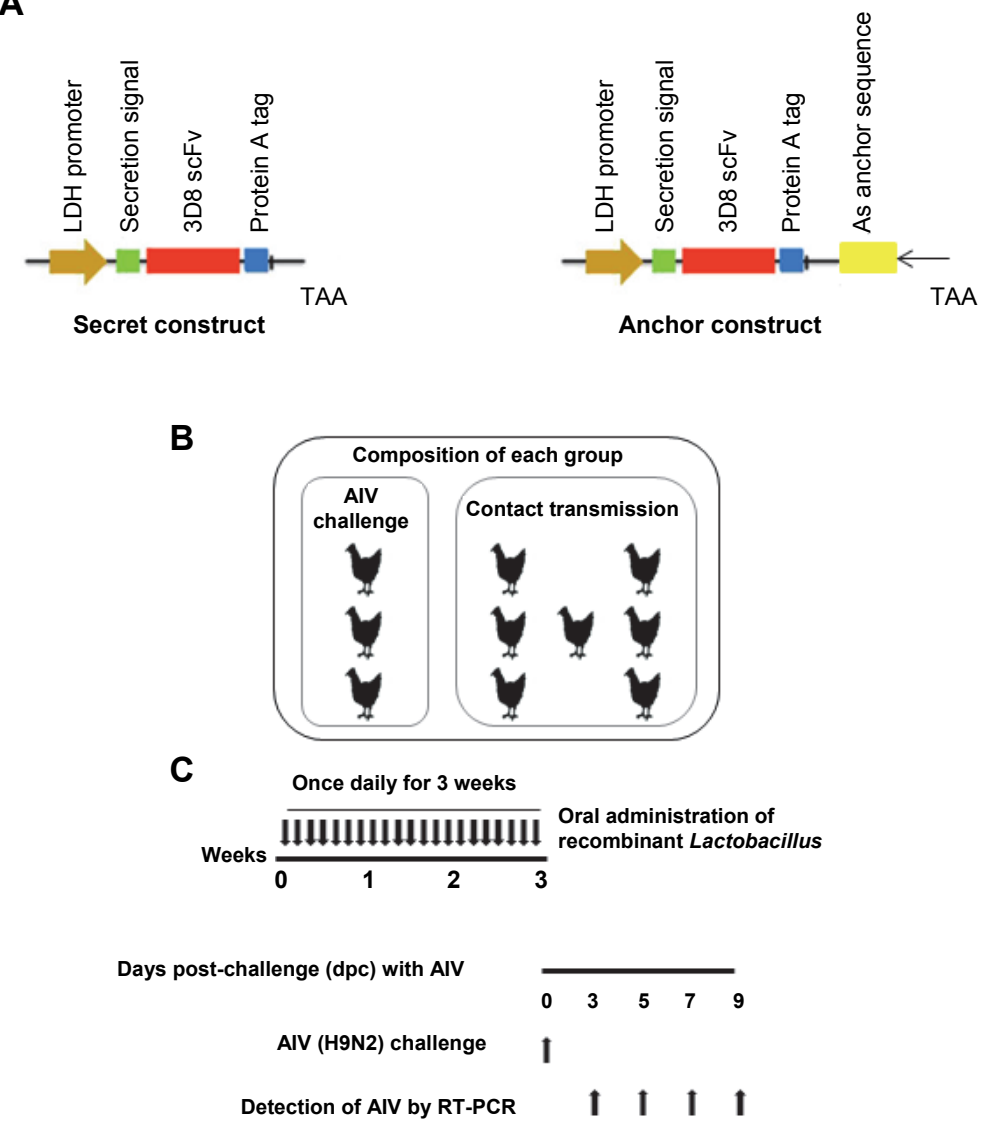

Fig. 1. Comparison of 3D8 scFv expression between $L$. paracasei expressing codon-optimised and the original sequence of $3 \mathrm{D} 8 \mathrm{scFv}(\mathrm{A})$ : Expression cassette designed to express $3 \mathrm{D} 8 \mathrm{scFv}$ as a secreted form (left) or anchored on the L. paracasei cell wall (right). Chickens were assigned to four groups, with 10 chickens per group. Three chickens were directly infected with $100 \mu \mathrm{L}$ of $10^{7.5} \mathrm{EID}_{50} / \mathrm{mL}$ H9N2 avian influenza virus (AIV challenge), and seven chickens were indirectly challenged (contact transmission) (B). Oropharyngeal and cloacal swabs samples were collected at $3,5,7$, and $9 \mathrm{~d}$ post-inoculation (dpi) from AIV-challenged chickens and contact transmission chickens $(\mathrm{C})$

\section{Statistical analysis}

Data analyses were conducted using GraphPad Prism statistical software (GraphPad Software). Challenge virus results were measured in oropharyngeal and cloacal swabs using RT-PCR, and the data were compared using one-way ANOVA and Tukey's tests. P values of less than 0.05 were considered statistically significant. Error bars denote standard deviation (SD). 


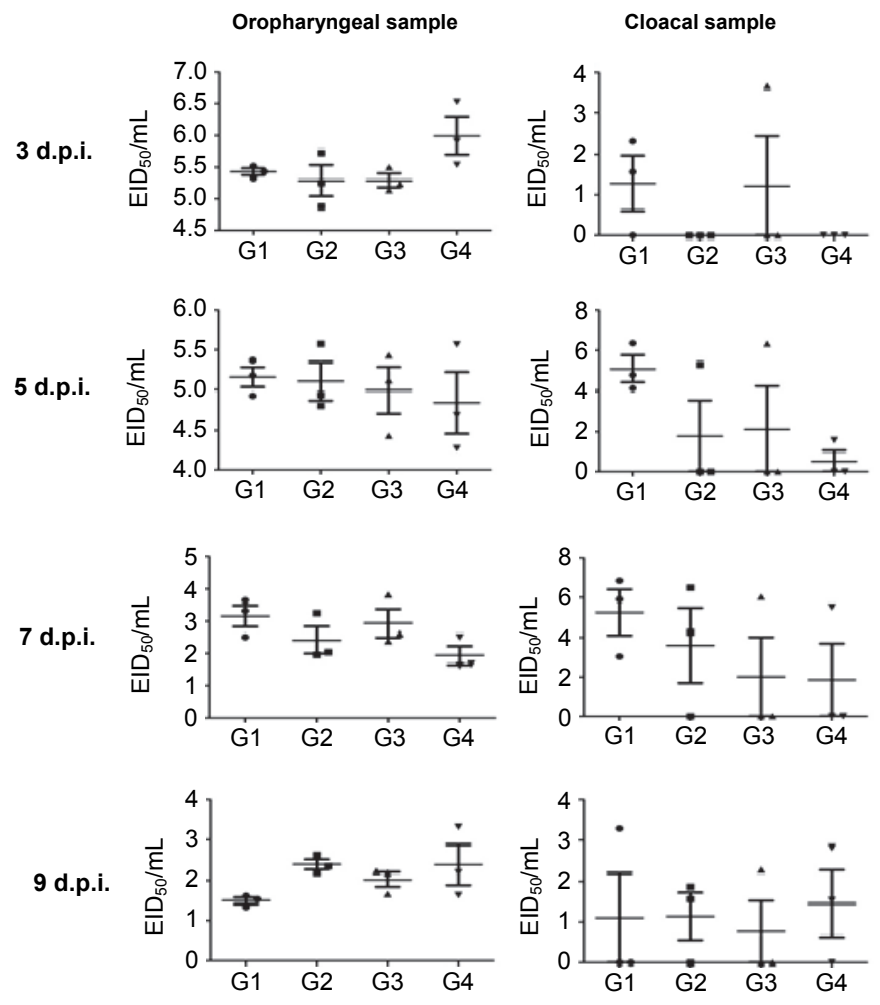

Fig. 2. Inhibition of AIV shedding levels after the oral administration of $L$. paracasei expressing the 3D8 in chicken oropharyngeal and cloacal swabs of the directly challenged group.

Three-week-old specific-pathogen-free (SPF) chickens were housed in each containment cage in a biosafety level 2 (BSL2) animal facility. Three chickens were intranasally challenged with $100 \mu \mathrm{L}$ of $10^{7.5} \mathrm{EID}_{50} / \mathrm{mL}$ H9N2 virus. Oropharyngeal and cloacal swab samples were harvested at $3,5,7$ and 9 days post-inoculation (dpi) from the challenged chickens and the viral RNA was quantified by real-time RT-PCR. Mean viral shedding titres were calculated. Data bars represent the mean \pm standard error

\section{Results and discussion}

In a previous study, our research team constructed the pSLp111.3 vector for expression in Lactobacillus provided by Dr. Jos Seegers (Falcobio, Netherlands). To induce 3D8 scFv protein expression, codon-optimised 3D8 $\mathrm{scFv}$ was cloned into pSLP-LDH that was slightly modified from the original vector as previously described (Hoang et al., 2015). Over the last two decades, the application of probiotics in the antiviral field has consisted primarily of anchoring viral antigens on the cell wall of probiotics in order to trigger an immune response (Seegers, 2002; Lee et al., 2006) or using probiotic-secreting proteins that act on cell surfaces to interrupt fusion between cellular and viral membranes (Rao et al., 
2005; Vangelista et al., 2010). In a different approach, in the present study, chickens were fed $10^{9}$ colony-forming units (CFU) of L. paracasei secreting 3D8 $\mathrm{scFv}$ or $L$. paracasei expressing cell wall-anchored 3D8 scFv using oral administration. We investigated the effect of oral administration of $3 \mathrm{D} 8 \mathrm{scFv}$-secreting L. paracasei using the experimental procedure shown in Fig. 1A. Each group included 10 chickens (H9N2 challenge: 3; contact transmission: 7) aged 3 weeks (Fig. 1B). After L. paracasei administration, the chickens were challenged with avian influenza virus. After challenge, individual chickens were monitored for 9 days. Oropharyngeal and cloacal swabs were collected at 3, 5, 7, and 9 dpi. The swabs were analysed by RT-PCR to detect AIV H9N2 (Fig. 1C).

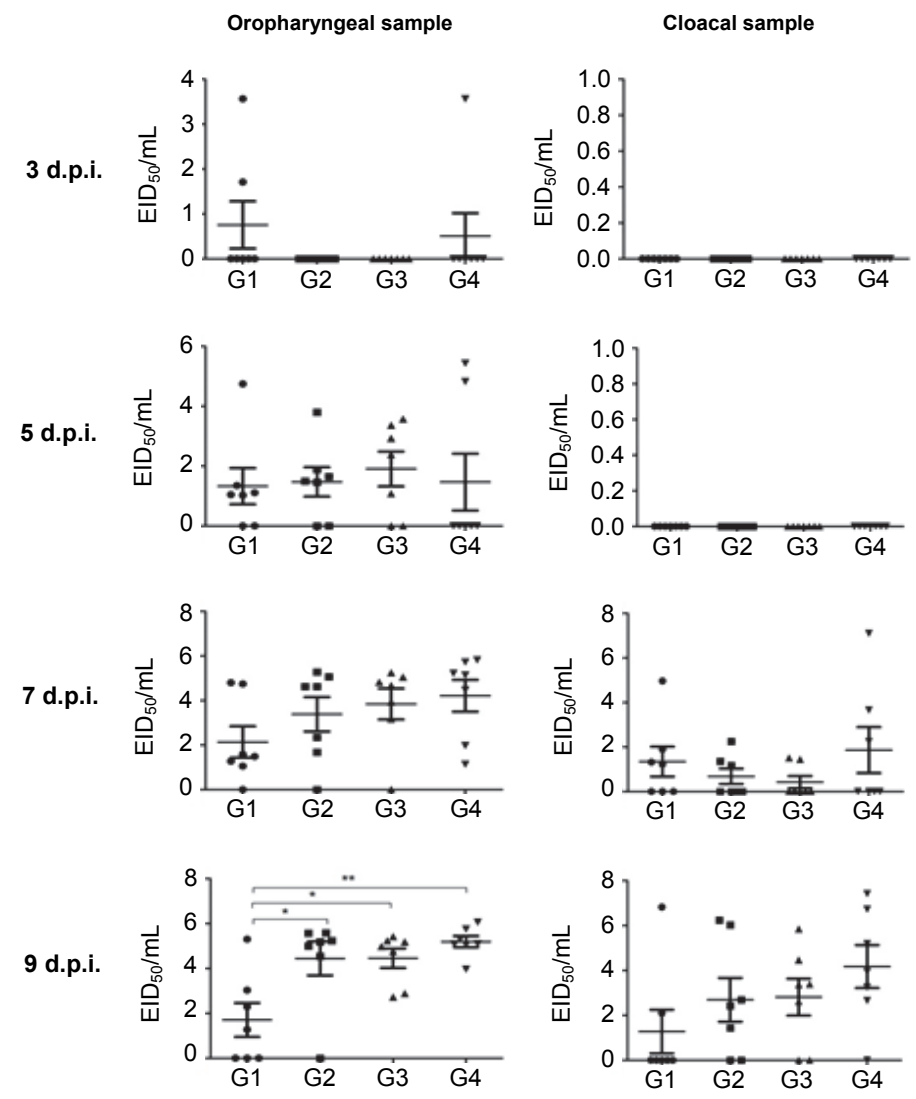

Fig. 3. Inhibition of the AIV shedding levels after the oral administration of $L$. paracasei expressing the 3D8 in chicken oropharyngeal and cloacal swabs of the contact exposed (indirectly challenged) group. Three-week-old specific-pathogen-free (SPF) chickens were housed in each containment cage in a biosafety level 2 (BSL2) animal facility. Seven chickens were intranasally challenged with $100 \mu \mathrm{L}$ of $10^{7.5} \mathrm{EID}_{50} / \mathrm{mL}$ H9N2 virus. Oropharyngeal and cloacal swab samples were harvested at 3, 5, 7 and 9 days post-inoculation (dpi) from the contact exposed chickens and the viral RNA was quantified by real-time RT-PCR. Mean viral shedding titres were calculated. Data bars represent the mean \pm standard error 
Rohani et al. (2009) reported that virus excretion mainly occurs through the oropharyngeal and cloacal routes, resulting in transmission not only by direct contact with infected hosts but also by indirect contact with infected hosts as well as indirectly through contaminated feeding grounds and surface waters. According to Byun et al. (2017), 3D8 scFv transgenic chickens exhibited reduced viral shedding levels in the contact transmission group in oropharyngeal and cloacal swab samples after challenge with avian influenza virus. In addition, Lee et al. (2019) reported that infectious bronchitis virus levels were significantly decreased in the $3 \mathrm{D} 8 \mathrm{scFv}$ transgenic chickens as compared to those in the contact transmission group. These findings were in agreement with our present research, in which $L$. paracasei-secreting 3D8 administration contact transmission chicken groups exhibited a significant reduction in viral shedding when compared with other groups.

The directly infected group showed similar viral RNA transcription level among the four treatment groups such as L. paracasei secreted $3 \mathrm{D} 8 \mathrm{scFv}, L$. paracasei anchored 3D8 scFv, L. paracasei, and the control group (Fig. 2). However, the group fed $3 \mathrm{D} 8 \mathrm{scFv}$-secreting $L$. paracasei had significantly reduced oropharyngeal virus shedding compared with the other three groups at 9 dpi (Fig. 3). In the present study, the group administered 3D8 scFv-secreting L. paracasei showed reduced viral shedding levels as compared to the contact transmission group. Previously Hoang et al. (2015) reported that codon optimisation also improved the expression of 3D8 scFv L. paracasei - secreting to reduce murine norovirus infection (MNV). Our findings also have important implications for characterising the antiviral properties of $3 \mathrm{D} 8 \mathrm{scFv}$-secreting L. paracasei in chickens. ScFvs such as 3D8 scFv that are formed by a single polypeptide are advantageous because of their small size, resistance to acid and heat, and ease of expression with an intact spatial structure. These properties make these scFvs suitable for the treatment of infections (Hoang et al., 2015). Although the mechanism of action of probiotics is not fully understood, the protective effect of the antibody-expressing $L$. paracasei in our system confirmed its antiviral activity. The advantages of using genetically modified $L$. paracasei include cost-efficient production, a long shelf life when lyophilised, simple logistics for distribution, and ease of administration (Pant et al., 2006; Hoang et al., 2015). In conclusion, the results obtained in the present study demonstrate that avian influenza virus transmission was suppressed in chickens fed L. paracasei expressing the 3D8 $\mathrm{scFv}$ protein. Therefore, the $3 \mathrm{D} 8 \mathrm{scFv}$ protein enhances probiotic activity against influenza virus transmission and is an effective antiviral protein that should be considered as an alternative treatment in the poultry industry. However, only few reports are available on the antiviral effect of the $3 \mathrm{D} 8 \mathrm{scFv}$ protein in chickens and, thus, further scientific investigations are needed. 


\section{Acknowledgements}

This study was supported by the 2015-2019 Postdoctoral Fellowship Program of the National Institute of Animal Science, Rural Development Administration, Republic of Korea. This work was conducted with the support of the 'Co-operative Research Program for Agriculture Science \& Technology Development (Project No. PJ01020101)' Rural Development Administration, Republic of Korea.

\section{References}

Byun, S. J., Yuk, S. S., Jang, Y. J., Choi, H., Jeon, M. H., Ochir, T. E., Kwon, J. H., Noh, J. Y., Kim, J. S., Yoo, J. G. and Song, C. S. (2017): Transgenic chickens expressing the 3D8 single chain variable fragment protein suppress avian influenza transmission. Sci. Rep. 7, 5938.

Dec, M., Wernicki, A., Puchalski, A. and Urban-Chmiel, R. (2015): Antibiotic susceptibility of Lactobacillus strains isolated from domestic geese. Brit. Poult. Sci. 56, 416-424.

Hoang, P. M., Cho, S., Kim, K. E., Byun, S. J., Lee, T. K. and Lee, S. (2015): Development of Lactobacillus paracasei harboring nucleic acid-hydrolyzing 3D8 scFv as a preventive probiotic against murine norovirus infection. Appl. Microbiol. Biotechnol. 99, 2793-2803.

Hoffmann, E., Stech, J., Guan, Y., Webster, R. and Perez, D. (2001): Universal primer set for the full-length amplification of all influenza A viruses. Arch. Virol. 146, 2275-2289.

Kang, H. K., Park, S. B. and Kim, C. H. (2016): Effect of dietary supplementation of red ginseng by-product on laying performance, blood biochemistry, serum immunoglobulin and microbial population in laying hens. Asian Australas. J. Anim. Sci. 29, 1464-1469.

Kim, Y. R., Kim, J. S., Lee, S. H., Lee, W. R., Sohn, J. N. and Chung, Y. C. (2006): Heavy and light chain variable single domains of an anti-DNA binding antibody hydrolyze both double- and single-stranded DNAs without sequence specificity. J. Biol. Chem. 281, 15287-15295.

Lee, G., Choi, H., Sureshkumar, S., Jung, S. K., Kim, J. S., Oh, K. B., Kim, K. W., Yang, H., Kim, D. H. and Byun, S. J. (2019): The 3D8 single chain variable fragment protein suppress infectious bronchitis virus transmission in the transgenic chickens. Res. Vet. Sci. 123, 293-297.

Lee, J. S., Poo, H., Han, D. P., Hong, S. P., Kim, K., Cho, M. W., Kim, E., Sung, M. H. and Kim, C. J. (2006): Mucosal immunization with surface-displayed severe acute respiratory syndrome coronavirus spike protein on Lactobacillus casei induces neutralizing antibodies in mice. J. Virol. 80, 4079-4087.

Lee, Y. N., Lee, D. H., Park, J. K., Lim, T. H., Youn, H. N. and Yuk, S. S. (2011): Isolation and characterization of a novel H9N2 influenza virus in Korean native chicken farm. Avian Dis. 55, 724-727.

Meimandipour, A., Hair-Bejo, M., Shuhaimi, M., Azhar, K., Soleimani, A. F. and Rasti, B. (2010): Gastrointestinal tract morphological alteration by unpleasant physical treatment and modulating role of Lactobacillus in broilers. Brit. Poult. Sci. 51, 52-59.

Oozeer, R., Furet, J. P., Goupil-Feuillerat, N., Anba, J., Mengaud, J. and Corthier, G. (2005): Differential activities of four Lactobacillus casei promoters during bacterial transit through the gastrointestinal tracts of human-microbiota-associated mice. Appl. Environ. Microbiol. 71, $1356-1363$.

Pant, N., Hultberg, A., Zhao, Y., Svensson, L., Pan-Hammarstrom, Q. and Johansen, K. (2006): Lactobacilli expressing variable domain of llama heavy-chain antibody fragments (lactobodies) confer protection against rotavirus-induced diarrhea. J. Infect. Dis. 194, 1580-1588.

Park, J. H., Lee, J. W., Choi, H., Jung, S. K., Kim, J. S., Kim, K. W., Oh, K. B., Yang, H. and Byun, S. J. (2018): Survival of Escherichia coli harboring nucleic acid-hydrolyzing 3D8 scFv during RNA virus infection. Reg. Tox. Pharmacol. 94, 286-292. 
Patel, S., Shukla, R. and Goyal, A. (2015): Probiotics in valorization of innate immunity across various animal models. J. Funct. Foods 14, 549-561.

Rao, S., Hu, S., McHugh, L., Lueders, K., Henry, K., Zhao, Q., Fekete, R. A., Kar, S., Adhya, S. and Hamer, D. H. (2005): Toward a live microbial microbicide for HIV: commensal bacteria secreting an HIV fusion inhibitor peptide. Proc. Natl Acad. Sci. USA 102, 11993-11998.

Ritzi, M. M., Abdelrahman, W., Mohnl, M. and Dalloul, R. A. (2014): Effects of probiotics and application methods on performance and response of broiler chickens to an Eimeria challenge. Poult. Sci. 93, 2772-2778.

Rohani, P., Breban, R., Stallknecht, D. E. and Drake, J. M. (2009): Environmental transmission of low pathogenicity avian influenza viruses and its implications for pathogen invasion. Proc. Natl Acad. Sci. USA 106, 10365-10369.

Seegers, J. F. (2002): Lactobacilli as live vaccine delivery vectors: progress and prospects. Trends Biotechnol. 20, 508-515.

Spackman, E., Senne, D., Bulaga, L., Myers, T., Perdue, M., Garber, P., Lohman, K., Daum, L.T. and Suarez, D. L. (2003): Development of real-time RT-PCR for the detection of avian influenza virus. Avian Dis. 47, 1079-1082.

Vangelista, L., Secchi, M., Liu, X., Bachi, A., Jia, L., Xu, Q. and Lusso, P. (2010): Engineering of Lactobacillus jensenii to secrete RANTES and a CCR5 antagonist analogue as live HIV-1 blockers. Antimicrob. Agents Chemother. 54, 2994-3001.

Wang, Z., Yu, Q., Fu, J., Liang, J. and Yang, Q. (2013): Immune responses of chickens inoculated with recombinant Lactobacillus expressing the haemagglutinin of the avian influenza virus. J. Appl. Microbiol. 115, 1269-1277.

Zhu, Y. Z., Cheng, J. L., Ren, M., Yin, L. and Piao, X. S. (2015): Effect of gamma-aminobutyric acidproducing Lactobacillus strain on laying performance, egg quality and serum enzyme activity in Hy-Line Brown hens under heat stress. Asian-Australas. J. Anim. Sci. 28, 1006-1013. 